\title{
Long-term Outcomes of Augmentation Enterocystoplasty in Patients With End-Stage Bladder Diseases: A Single-Institute Experience Involving 102 Patients
}

\author{
Shu-Yu Wu, Yuan-Hong Jiang, Hann-Chorng Kuo \\ Department of Urology, Buddhist Tzu Chi General Hospital, Tzu Chi University, Hualien, Taiwan
}

Purpose: Augmentation enterocystoplasty (AE) has been shown to improve clinical symptoms in patients with end-stage bladder disease (ESBD). Herein, we report the long-term outcomes of a series of patients with different etiologies of ESBD who received $\mathrm{AE}$.

Methods: We retrospectively reviewed 102 patients with ESBD who received AE at the Hualien Tzu Chi General Hospital from 1992 to 2014. ESBD in this study was defined as including neurogenic lower urinary tract dysfunction (NLUTD) due to spinal cord injury (SCI) or myelomeningocele, inflammatory bladder disease (IBD), ESBD occurring after pelvic cancer surgery, and other etiologies. Complications including active lower urinary tract problems and urinary tract infection (UTI), as well as patients' self-reported satisfaction with the procedure, were evaluated.

Results: A total of 102 patients were included in the study. A majority of patients received AE for NLUTD $(n=43)$, followed by $\operatorname{IBD}(n=38)$, ESBD after pelvic cancer surgery $(n=15)$, and the other etiologies $(n=6)$. Patients had a mean age of $39.4 \pm 18.7$ years and were followed for a mean of 78 months. All patients had significantly increased cystometric bladder capacity and compliance at the time of follow-up. Fifty-four patients (52.9\%) reported moderate to excellent satisfaction with the outcome, and there were no significant differences among the groups $(\mathrm{P}=0.430)$. The most common reason for dissatisfaction was the need for clean intermittent catheterization (CIC; 41.7\%), followed by urinary incontinence (25.0\%) and recurrent UTI (16.7\%).

Conclusions: AE is a safe and effective procedure for patients with ESBD. Postoperative urinary incontinence and UTI as well as the need for CIC may affect quality of life and decrease patient satisfaction.

Keywords: Lower Urinary Tract Symptoms; Urinary Bladder Diseases; Cystitis; Urinary Bladder, Neurogenic

- Research Ethics: This study was approved by the Ethics Committee of Buddhist Tzu Chi General Hospital, Hualien, Taiwan (approval number: IRB105-69-B). Informed consent was waived by the Ethics Committee of the Buddhist Tzu Chi General Hospital, Hualien, Taiwan, as the chart review involved a regular treatment and the study was retrospectively performed.

- Conflict of Interest: No potential conflict of interest relevant to this article was reported.

\section{INTRODUCTION}

Irreversible contracted bladder and poor bladder compliance due to different diseases can be categorized as end-stage blad- der disease (ESBD) [1]. Poor bladder compliance leads to further upper urinary tract damage and can result in renal function impairment. Several studies have shown that a contracted bladder can result in bilateral hydronephrosis or acute renal
Corresponding author: Hann-Chorng Kuo (iD https://orcid.org/0000-0001-7165-4771 Department of Urology, Buddhist Tzu Chi General Hospital, 707 Chung-Yang Road, Section 3, Hualien 970, Taiwan

E-mail: nobookrain2014@gmail.com; hck@tzuchi.com.tw /

Tel \& Fax: +88-638651825 (ext. 2113)

Submitted: August 26, 2016 / Accepted after revision: November 10, 2016 
failure [2-4]. Emergency percutaneous nephrostomy diversion is required in some patients. Contracted bladder can occur due to spinal cord injury (SCI), myelomeningocele (MMC), inflammatory bladder disease (IBD), or after radical surgery for pelvic cancer.

Augmentation enterocystoplasty (AE) has been shown to be effective in improving clinical symptoms associated with ESBD [1]. Enterocystoplasty is indicated in patients with anatomically reduced bladder capacity and compliance due to neurogenic bladder, tuberculosis, radiation cystitis, or ulcer-type interstitial cystitis [5]. The procedure has been used for treating bladder dysfunction for more than 100 years, and much of our knowledge of the complications comes from pediatric patients with MMC [6]. Enterocystoplasty is usually performed to lower bladder storage pressure and increase bladder capacity in patients with medically refractory disease. However, the shortterm and long-term complication rates associated with enterocystoplasty remain high and are potentially serious. Therefore, some centers have begun earlier and more aggressive medical therapy in an attempt to avoid the need for the procedure [7]. Enterocystoplasty is also used in the adult population for neurogenic bladder dysfunction as well as for an overactive bladder, IBD that results in a severely contracted bladder, ulcer-type interstitial cystitis, and reconstruction for iatrogenic bladder damage as a result of the treatment of bowel and gynecological malignancies [8].

Enterocystoplasty is a procedure with stable long-term outcomes and high rates of patient satisfaction [9]. However, no studies have investigated the outcomes and complications of the procedure associated with different etiologies of ESBD. Herein, we report the long-term results of a large series of patients with $\mathrm{ESBD}$ who received $\mathrm{AE}$ at a single institute.

\section{MATERIALS AND METHODS}

\section{Patients}

We retrospectively reviewed the medical and surgical records of all patients who received AE for irreversible ESBD due to different etiologies at the Hualien Tzu Chi General Hospital from 1992 to 2014. All patients presented with elevated urinary frequency, incontinence, upper urinary tract dysfunction, or intolerable bladder pain before surgery; further, all of them underwent the procedure because their conditions were refractory to conservative treatment.

A detailed history was taken, and physical examinations were performed for all patients. Videourodynamic studies, cystoscopy, and ultrasound of the upper urinary tract were performed in all the patients before $\mathrm{AE}$ and at their most recent follow-up. The data from the most recent studies were used for a postoperative analysis. Bladder capacity was determined by a urodynamic study (cystometric bladder capacity [CBC]) or the maximal functional volume from self-voiding or catheterization (functional bladder capacity [FBC]). The presence of vesicoureteral reflux (VUR) was confirmed by the videourodynamic study. All patients who underwent AE had low CBC and FBC and low bladder compliance with or without VUR.

The patients were classified into 4 subgroups according to the ESBD etiology, namely neurogenic lower urinary tract dysfunction (NLUTD; due to SCI or MMC), IBD (such as ulcertype interstitial cystitis, ketamine cystitis, tuberculosis cystitis, and eosinophilic cystitis), a sequela of pelvic cancer surgery (such as cervical cancer and colon cancer but not bladder cancer), and other etiologies, such as multiple sclerosis, cerebral palsy, and systemic lupus erythematosus (SLE)-induced contracted bladder.

\section{Surgical Procedures}

AE was performed under general anesthesia by a single surgeon using a terminal ileal segment based on the modified Hartmann procedure $[4,10]$. A $40-\mathrm{cm}$ terminal ileal loop was selected $30 \mathrm{~cm}$ from the ileocecal valve after appendectomy. If ureter reimplantation was needed, the ureters were dissected until the vascular bifurcation was reached. The speculated ureters were anastomosed at the end of the troughs of the detubularized ileum to form an extramural serosa-lined tunnel. Ureteral reimplantation was performed in patients with high-grade VUR according to the videourodynamic study before the operation.

The entire segment of the resected ileum was transformed into an " $M$ " shape by suturing the seromuscular layer $2 \mathrm{~cm}$ from the medial edges of the flaps of the ileum, followed by detubularization by opening along the antimesenteric borders. The ileal pouch was made under a double layer suture of the intestinal serosa with 3-0 vicryl on the inner layer and 3-0 silk on the outside. The native contracted bladder was opened transversely as wide as possible to create a clam-shape bladder. For patients with ulcer-type interstitial cystitis partial cystectomy was performed to excise the bladder lesion. A small segment of the bladder was sent for a pathology evaluation. The fashioned ileal patch was then anastomosed to the bladder wall by using double-layer 3-0 vicryl sutures. 
After the anastomosis, a large 3-way Foley catheter (usually 22F) was inserted to allow for continuous bladder irrigation with normal saline. Two large Jackson-Pratt drains were placed at the intestinal anastomotic site and the perivesical space.

Continuous bladder irrigation was performed for 1 day after the operation, and then, intermittent bladder irrigation was performed every day for 3 days after the operation to prevent intestinal mucus-induced catheter obstruction. The patients were administered postoperative short-term pain control medications until discharge. The Foley catheter was kept in place for at least 10-14 days after the operation and was removed when cystography showed no evidence of extravasation. If patients could not void spontaneously or by abdominal straining after removal of the catheter, then they were taught how to perform clean intermittent catheterization (CIC).

\section{Follow-up}

Postoperative follow-up included a regular videourodynamic study and renal and bladder ultrasonography. The videourodynamic study was performed about 1 to 3 months after the operation and then, annually if patients returned for follow-up. Patients also received ultrasonography at every clinical follow-up. Postoperative cystoscopy was also performed for specific indi- cations including hematuria, refractory incontinence, or recurrent UTI. UTI was defined in patients who had positive urine or blood culture test results after admission for a febrile episode. The global response assessment (GRA) was used for measuring patient satisfaction with the surgical outcome. In this study, the GRA measure was graded on a 4-point Likert scale as follows: 0 , not satisfied; 1 , mildly satisfied; 2 , moderately satisfied; and 3, very satisfied. Patients with exacerbated symptoms were also graded as not satisfied [11].

\section{Statistical Analysis}

The urodynamic parameters, rates of CIC, and patient satisfaction were compared among subgroups with the paired $\mathrm{t}$-test for a longitudinal comparison. Analysis of variance and post hoc analyses were used for subgroup comparisons. P-values $<0.05$ were considered to indicate statistical significance. All analyses were performed with SPSS ver. 12 (SPSS Inc., Chicago, IL, USA).

\section{RESULTS}

A total of 102 patients (55 men and 47 women) were included in this study. The mean age was $39.4 \pm 18.7$ years, and the mean follow-up period was 78 months (range, 12-122 months).

Table 1. Baseline and follow-up results in the end-stage bladder disease patients received augmentation enterocystoplasty

\begin{tabular}{|c|c|c|c|c|c|c|}
\hline Variable & $\begin{array}{l}\text { Overall } \\
(\mathrm{n}=102)\end{array}$ & $\begin{array}{l}\text { NLUTD } \\
(\mathrm{n}=43)\end{array}$ & $\begin{array}{c}\text { IBD } \\
(n=38)\end{array}$ & $\begin{array}{l}\text { Post pelvic cancer } \\
\text { surgery }(n=15)\end{array}$ & $\begin{array}{l}\text { Others } \\
(n=6)\end{array}$ & P-value \\
\hline \multicolumn{7}{|l|}{$\mathrm{CBC}(\mathrm{mL})$} \\
\hline Baseline & $107 \pm 78.5$ & $117 \pm 57.6$ & $54.5 \pm 33.7$ & $173 \pm 125$ & $190 \pm 66.6$ & $<0.001$ \\
\hline Follow-up & $425 \pm 133^{\mathrm{a})}$ & $499 \pm 91.2^{\mathrm{a})}$ & $324 \pm 77.4^{\mathrm{a})}$ & $385 \pm 149^{\mathrm{a})}$ & $541 \pm 204^{\text {a) }}$ & $<0.001$ \\
\hline \multicolumn{7}{|l|}{ Voided volume (mL) } \\
\hline Baseline & $55.8 \pm 61.8$ & $44.8 \pm 35.0$ & $44.5 \pm 32.8$ & $115 \pm 129$ & $66.4 \pm 47.4$ & 0.007 \\
\hline Follow-up & $170 \pm 498^{\mathrm{a})}$ & $113 \pm 172^{a)}$ & $226 \pm 137^{a)}$ & $226 \pm 176$ & $149 \pm 174$ & 0.100 \\
\hline \multicolumn{7}{|l|}{ PVR (mL) } \\
\hline Baseline & $52.3 \pm 64.9$ & $72.9 \pm 64.3$ & $9.4 \pm 19.5$ & $60.0 \pm 81.1$ & $134 \pm 55.0$ & $<0.001$ \\
\hline Follow-up & $227 \pm 233^{a)}$ & $431 \pm 195^{a)}$ & $104 \pm 119^{\mathrm{a})}$ & $145 \pm 143$ & $442 \pm 307$ & $<0.001$ \\
\hline \multicolumn{7}{|l|}{ Compliance $\left(\mathrm{mL} / \mathrm{cm} \mathrm{H}_{2} \mathrm{O}\right)$} \\
\hline Baseline & $9.6 \pm 39.0$ & $7.2 \pm 4.9$ & $13.7 \pm 22.1$ & $7.4 \pm 6.9$ & $10.2 \pm 4.8$ & 0.004 \\
\hline Follow-up & $56.3 \pm 61.8^{\mathrm{a})}$ & $63.3 \pm 36.9^{\mathrm{a})}$ & $53.6 \pm 43.9^{\mathrm{a})}$ & $43.0 \pm 35.0^{a)}$ & $50.0 \pm 32.1^{\mathrm{a})}$ & 0.269 \\
\hline Need of CIC (\%) & 48.00 & 76.70 & 15.80 & 40.00 & 66.70 & $<0.001$ \\
\hline Urinary incontinence, n (\%) & $14(13.7)$ & $9(20.9)$ & $2(5.3)$ & $3(20.0)$ & $0(0)$ & 0.133 \\
\hline Satisfaction (\%) & 52.90 & 53.50 & 60.50 & 40.00 & 33.30 & 0.430 \\
\hline UTI > 1/yr (\%) & 34.30 & 32.60 & 34.20 & 40.00 & 33.30 & 0.966 \\
\hline
\end{tabular}

Values are presented as mean \pm standard deviation unless otherwise indicated.

NLUTD, neurogenic lower urinary tract dysfunction; IBD, inflammatory bladder disease; CBC, cystometric bladder capacity; PVR, postvoid residual urine; CIC, clean intermittent catheterization; UTI, urinary tract infection.

${ }^{a}$ Significant difference compared with baseline. 
Overall, the CBC, bladder compliance, self-voided volume, and postvoid residual (PVR) urine volume increased significantly after AE (Table 1). At the follow-up, 48 patients (47.1\%) had spontaneous voiding without the need for urethral catheterization, 49 (48\%) had to perform CIC, and 5 (5\%) chose to keep the indwelling urethral catheter.

All patients with NLUTD ( $n=43), \operatorname{IBD}(n=38)$, ESBD due to pelvic cancer surgery $(\mathrm{n}=15)$, and ESBD due to other etiologies $(\mathrm{n}=6)$ had significantly higher $\mathrm{CBC}$ and increased bladder compliance at the follow-up (Table 1). Among the 29 patients who presented with VUR at the baseline, 23 received ureteral reimplantation during $\mathrm{AE}$, and of them, only 2 (6.9\%) still had VUR at the follow-up. CIC had to be performed in 33 patients with NLUTD (76.7\%), 6 (40\%) with ESBD due to pelvic cancer surgery, 4 (66.7\%) with ESBD due to other etiologies, and 6 (15.8\%) with IBD. Further, significant differences were observed among the groups $(\mathrm{P}<0.001)$. In the 49 patients who performed CIC, 15 (30.6\%) were only partially dependent on CIC. The other 34 patients (69.4\%) were completely dependent on CIC, including 26 in the NLUTD group, 5 with preoperative detrusor acontractility, and 3 with other etiologies. Five patients with NLUTD chose to keep the indwelling urethral catheter because they felt that it was inconvenient to perform CIC during working hours.

Of the 102 patients, 54 (52.9\%) reported moderate to excellent satisfaction. There were no significant differences in the degree of satisfaction among the patient subgroups $(\mathrm{P}=0.430)$ (Table 1). Patients in the IBD group had the highest satisfaction rate $(60.5 \%)$. The most common reason for dissatisfaction was the need for CIC (41.7\%), followed by urinary incontinence (25.0\%) and recurrent UTI (16.7\%). The rate of CIC was significantly lower in patients with IBD and was significantly higher in patients with NLUTD. The intractable bladder pain usually resolved immediately after the operation. Therefore, patients with interstitial cystitis who received AE did not complain of pain after the operation; instead, they all reported good satisfaction because they were pain-free. However, difficult urination, the need for CIC, and recurrent UTI remained the main causes of dissatisfaction.

Urinary incontinence was a bothersome adverse event after $\mathrm{AE}$ in our patients. Although urinary incontinence was common during the first few postoperative months, most patients could achieve continence after 6 months. Before the operation, urodynamic detrusor overactivity (DO) was detected in 35 patients (34.3\%); most of them belonged to the IBD group. Only 5 of them (4.9\%) had persistent DO after AE. However, nocturnal urinary incontinence was reported by $13.7 \%$ of the patients who had low urethral resistance and could only void spontaneously by using the Crede maneuver, including 9 NLUTD patients, 2 IBD patients, and 3 pelvic cancer surgery patients. None of the patients who needed CIC reported having nocturnal urinary incontinence.

Most patients (65.7\%) had less than one UTI episode per year, $30.4 \%$ had 1 to 3 episodes per year, and 3.9\% of the patients had more than 3 UTI episodes per year. There were no significant differences in the UTI frequency among the subgroups. Patients who used CIC or required indwelling urethral catheters had a higher rate of recurrent UTI $(\mathrm{P}=0.039)$. Bladder stones developed in 5 patients (4.9\%). Two patients with IBD developed enterovesical anastomosis stricture, resulting in difficulty with bladder emptying at the time of follow-up. In both patients, the stricture was successfully treated with a transurethral incision of the stricture ring. No patient reported organ perforation or new-onset malignancy at the time of follow-up.

\section{DISCUSSION}

The results of this study showed that AE could significantly improve bladder capacity and compliance in patients with ESBD caused by different etiologies. There were no differences in satisfaction or complication rates between the patient subgroups. CIC was necessary in most patients with NLUTD and was the major cause of dissatisfaction. Recurrent UTI was a common complication of AE, particularly in patients who had to perform CIC or required indwelling urethral catheters.

There are a number of causes of ESBD, including neurogenic causes, such as SCI and MMC, and nonneurogenic causes, such as IBD and ESBD as a side effect of radical surgery for pelvic cancer. ESBD may result in reduced bladder compliance and may further lead to upper urinary tract damage. Emergency treatment may be needed in some patients to prevent acute renal failure.

The goals of managing reduced bladder capacity or poor bladder compliance include protecting renal function, maximizing continence, minimizing urological morbidity, and improving the patient's quality of life. Patients with bladder dysfunction refractory to conservative measures (behavioral, lifestyle modification, and drug and intravesical therapies) are candidates for enterocystoplasty [5]. More commonly, the indications are extended to include socially unacceptable inconti- 
nence due to small bladder capacity, refractory DO, and decreased compliance [12,13].

Many studies have investigated the outcome of $\mathrm{AE}$ in patients with neurogenic disorders such as traumatic SCI, MMC, and spinal bifida [10,12-14]. However, the outcomes of AE in patients with non-neurogenic disorders have rarely been studied. In this study, we included patients with nonneurogenic causes of ESBD, such as ulcer-type interstitial cystitis and ketamine cystitis, diseases that are currently considered to be contraindications for enterocystoplasty [1].

We found that voiding parameters including $\mathrm{CBC}$, voiding volume, PVR urine volume, and bladder compliance improved significantly after AE in every group, and that a majority of patients $(52.9 \%)$ reported moderate to excellent satisfaction with the outcome. Patients with IBD, particularly those who did not require CIC, had the highest rate of satisfaction, while those with ESBD due to other etiologies were the least satisfied. Nonetheless, there were no significant differences in satisfaction rates among the subgroups. CIC was the major reason for dissatisfaction in our study. Patients who had received prior surgery for pelvic cancer, those with multiple sclerosis, and patients with cerebral palsy or SLE had the lowest satisfaction rates, possibly because these patients did not show a significant change in the voided volume and because the majority required CIC.

In this study, we found that increased bladder capacity after $\mathrm{AE}$ was associated with an increase in the postoperative PVR urine volume. Of the 102 patients considered in this study, 48\% $(n=49)$ needed to perform CIC after the operation and most of these patients underwent AE for NLUTD. The reason for the high rate of CIC usage among patients with NLUTD is most likely the fact that the etiology of NLUTD in a majority of patients was neurogenic in nature (SCI or MMC), which impaired their bladder sensation or decreased their voiding efficiency, thereby making it necessary for them to perform CIC after AE. In addition, patients who developed ESBD after pelvic surgery and patients with ESBD due to other etiologies had detrusor underactivity at baseline, making spontaneous voiding through the urethra impossible and necessitating postoperative CIC. Preoperative video urodynamics might be helpful in predicting the need for postoperative $\mathrm{CIC}$ in patients who require $\mathrm{AE}$.

Other common causes of patient dissatisfaction were urinary incontinence and UTI. A previous study showed that $8 \%$ of the patients required subsequent incontinence procedures and that $5 \%$ required further urinary diversion after $\mathrm{AE}$ [14]. In our study, the postoperative incontinence rate was $13.7 \%$; all of the affected patients reported urgency incontinence or stress urinary incontinence. No statistically significant differences were observed according to etiology. Urinary incontinence usually occurred at night, and most of the patients reported being able to void spontaneously using the Crede maneuver. Patients who were completely dependent on CIC to empty their bladder did not have this adverse event. During the first few postoperative months, active intestinal peristaltic waves might overcome urethral resistance and cause urinary incontinence. At 6 months after $\mathrm{AE}$, the peristaltic pressure decreases and bladder compliance improves. Peristaltic wave pressure in augmented intestines has been reported to be less than $20 \mathrm{~cm} \mathrm{H}_{2} \mathrm{O}$ [15]. The low intravesical pressure usually does not exceed the urethral resistance, thereby increasing urinary continence. However, patients who can void by abdominal straining might have lower urethral resistance, which can lead to nocturnal urinary incontinence. In our study, the degree of incontinence was mild and a majority of patients could manage this adverse event by wearing a night pad or decreasing their liquid intake before sleep. None of the patients required further surgical intervention.

Bacteriuria is common after AE. CIC is a well-known route for bacterial colonization, and the altered bladder surface, presence of mucus, and incomplete bladder emptying via the catheter can promote bacterial growth. Treatment is typically indicated only for symptomatic cases of UTI, including new-onset incontinence, suprapubic pain, hematuria, foul-smelling urine, or fever, or for urea-splitting organisms that may contribute to bladder stone formation $[16,17]$. We found an overall high incidence of recurrent UTI (34.3\%) in this study, and there were no significant differences in the incidence among the subgroups. This finding might be attributed to the fact that there were similar percentages of patients in each subgroup that emptied their bladders via abdominal straining, urethral catheters, or CIC usage.

The major limitations of this study are the differences in case number between subgroups and the use of self-reported grading for outcome satisfaction. Although $48 \%$ of the patients with ESBD were not satisfied with the surgical outcome, most of them did not develop the urological complications that commonly arise from a contracted bladder and poor bladder compliance. Patients with ESBD desire to have a large, low-pressure bladder after AE. They also expect to void spontaneously without the aid of abdominal pressure or a catheter. In fact, the possible outcomes of surgery might not meet their high expectations, resulting in a relatively low satisfaction rate. With this un- 
derstanding, patients should be informed about the possible surgical outcomes before $\mathrm{AE}$ is performed for ESBD.

$\mathrm{AE}$ for patients with ESBD is a safe and effective procedure and achieves satisfactory subjective and objective outcomes. The postoperative needs for CIC, urinary incontinence, and the development of UTI may affect a patient's quality of life and decrease patient satisfaction. Patients should be well informed of the risks before undergoing $\mathrm{AE}$ and receive long-term followup after surgery.

\section{REFERENCES}

1. Blaivas JG, Weiss JP, Desai P, Flisser AJ, Stember DS, Stahl PJ. Longterm followup of augmentation enterocystoplasty and continent diversion in patients with benign disease. J Urol 2005;173:1631-4.

2. Oelke M, Höfner K, Wiese B, Grünewald V, Jonas U. Increase in detrusor wall thickness indicates bladder outlet obstruction (BOO) in men. World J Urol 2002;19:443-52.

3. Chu PS, Ma WK, Wong SC, Chu RW, Cheng CH, Wong S, et al. The destruction of the lower urinary tract by ketamine abuse: a new syndrome? BJU Int 2008;102:1616-22.

4. Chen JL, Kuo HC. Long-term outcomes of augmentation enterocystoplasty with an ileal segment in patients with spinal cord injury. J Formos Med Assoc 2009;108:475-80.

5. Mitrofanoff P. Trans-appendicular continent cystostomy in the management of the neurogenic bladder. Chir Pediatr 1980;21:297305.

6. Lendvay TS, Cowan CA, Mitchell MM, Joyner BD, Grady RW. Augmentation cystoplasty rates at children's hospitals in the United States: a pediatric health information system database study. J Urol
2006;176(4 Pt 2):1716-20.

7. Edelstein RA, Bauer SB, Kelly MD, Darbey MM, Peters CA, Atala $\mathrm{A}$, et al. The long-term urological response of neonates with myelodysplasia treated proactively with intermittent catheterization and anticholinergic therapy. J Urol 1995;154:1500-4.

8. Reyblat P, Ginsberg DA. Augmentation cystoplasty: what are the indications? Curr Urol Rep 2008;9:452-8.

9. $\mathrm{Ku} \mathrm{JH}$. The management of neurogenic bladder and quality of life in spinal cord injury. BJU Int 2006;98:739-45.

10. Hautmann RE, Egghart G, Frohneberg D, Miller K. The ileal neobladder. J Urol 1988;139:39-42.

11. Propert KJ, Mayer RD, Wang Y, Sant GR, Hanno PM, Peters KM, et al. Responsiveness of symptom scales for interstitial cystitis. Urology 2006;67:55-9.

12. Scales CD Jr, Wiener JS. Evaluating outcomes of enterocystoplasty in patients with spina bifida: a review of the literature. J Urol 2008; 180:2323-9.

13. Welk B, Herschorn S, Law C, Nam R. Population based assessment of enterocystoplasty complications in adults. J Urol 2012;188:464-9.

14. Hendren WH, Hendren RB. Bladder augmentation: experience with 129 children and young adults. J Urol 1990;144(2 Pt 2):445-53.

15. Kuo HC. Clinical outcome and quality of life after enterocystoplasty for contracted bladders. Urol Int 1997;58:160-5.

16. Herschorn S, Hewitt RJ. Patient perspective of long-term outcome of augmentation cystoplasty for neurogenic bladder. Urology 1998; 52:672-8.

17. Shekarriz B, Upadhyay J, Demirbilek S, Barthold JS, González R. Surgical complications of bladder augmentation: comparison between various enterocystoplasties in 133 patients. Urology 2000;55: 123-8. 